Accepted: 12 May 2012

Received: 19 March 2012

Volume: 1

Number: 1

Page: 17 - 26

May 2012
Pub. Online: 10 July 2012

\section{Modelling Very Long Baseline Interferometry (VLBI) observations}

\author{
Kamil Teke ${ }^{1, *}$, Emine Tanır Kayıkçı ${ }^{2}$, Johannes Böhm ${ }^{3}$ and Harald Schuh ${ }^{3}$ \\ ${ }^{1}$ Hacettepe University, Faculty of Engineering, Geomatics Engineering Department, 06800, Ankara, Turkey \\ ${ }^{2}$ Karadeniz Technical University, Faculty of Engineering, Geomatics Engineering Department, 61080, Trabzon, Turkey \\ ${ }^{3}$ Vienna University of Technology, Institute of Geodesy and Geophysics, 1040, Vienna, Austria
}

\section{Abstract}

The main objective of this study is to provide in detail the theoretical basis of the Very Long Baseline Interferometry (VLBI) delay model, mainly according to the International Earth Rotation and Reference Systems Service (IERS) Conventions 2010. This goes along with introducing the concept of continuous piece-wise linear offset (CPWLO) functions for estimating sub-daily geodetic parameters at pre-defined epochs, e.g. at Universal Time (UT) integer hours or at integer fractions or multiples of integer hours. The geodetic parameters can be simultaneously and accurately estimated from VLBI observations in sub-daily resolution if enough observations within each estimation interval are carried out from homogenously distributed Earth-fixed VLBI antennas to space-fixed radio sources. After providing the basic VLBI model of the geometric delay including clock synchronization and tropospheric effects, the partial derivatives of VLBI observation equation with respect to the most important geodetic parameters are given and some typical VLBI results are shown.

Keywords

Very Long Baseline Interferometry (VLBI), VLBI delay model, continuous piece-wise linear offsets, CPWLO.

\section{Özet}

\section{Çok Uzun Baz Enterferometrisi (VLBI) ölçülerinin modellenmesi}

Kabul: 12 Mayıs 2012

Alındı: 19 Mart 2012

Bu çalışmanın temel amacı Çok Uzun Baz Enterferometrisi (VLBI) sinyal gecikme modelinin teorik detaylarını Uluslararası Yer Dönüklük ve Referans Sistemleri Servisi (IERS) 2010 Konvansiyonları temelinde sun-

Web Yayın: 10 Temmuz 2012

Cilt: 1

Sayı: 1

Sayfa: $17-26$

Mayıs 2012 maktır. Bu kapsamda sürekli parçalı lineer offset (CPWLO) fonksiyonları ile gün-içi zamansal çözünürlükte önceden belirlenmiş epoklarda, örneğin: Evrensel Zaman (UT) tam saatleri, tam saatlerin katlarl veya bölümlerinde jeodezik parametrelerin kestirimi ayrıca açılkanmıştır. Yerküre'deki homojen dağılımlı Yer-sabit VLBI antenlerinden gök küredeki homojen dağllımlı uzay-sabit radyo kaynaklarına her parametre kestirim epoğu aralığında yapılacak yeterli sayıda VLBI ölçülerinden, jeodezik parametreler gün-içi zamansal çözünürlükte eşzamanlı ve duyarlı̆̆ yüksek olarak kestirilebilir. Saat senkronizasyon ve troposfer gecikme modellerini içeren temel VLBI gecikme modeli sunulduktan sonra VLBI ölçü denkleminin başlıca jeodezik parametrelere göre kısmi türevleri verilmiş ve tipik VLBI sonuçları gösterilmiştir.

Anahtar Sözcükler

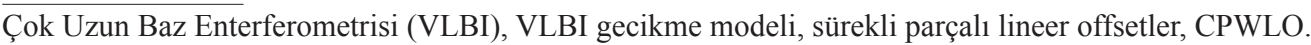




\section{Introduction}

Very Long Baseline Interferometry (VLBI) is a part of geodesy for nearly 40 years. Since 1999 VLBI is coordinated by the International VLBI Service for Geodesy and Astrometry (IVS) - a Service of the International Association of Geodesy (IAG) and International Astronomical Union (IAU) (Schlüter and Behrend 2007). The main data and products that VLBI technique provides for scientific community are; Celestial Intermediate Pole (CIP) position in Earth-fixed reference system i.e. International Terrestrial Reference System (ITRS) (polar motion coordinates), the Earth axis absolute rotation angle w.r.t. space-fixed reference system i.e. Geocentric Celestial Reference System (GCRS), the CIP position in ICRS (nutation offsets), source coordinates in Barycentric Celestial Reference System (BCRS), antenna Cartesian coordinates in ITRF, ionosphere delays, elevation angle dependent troposphere delays, delays due to azimuthal asymmetric parts of the troposphere (gradients), clock errors, special geophysical parameters such as tidal Love and Shida numbers and several technique specific parameters.

Very Long Baseline Interferometry (VLBI) is a geometric space geodetic technique: it measures the time difference between the arrival of a radio wavefront emitted by a distant quasi-stellar radio source (quasar) at two Earth-based antennas (Figure 1).

Using large numbers of time difference measurements from many quasars observed with a global network of antennas, VLBI determines the inertial reference frame (spacefixed frame, e.g. ICRF2 (Fey et al. 2009) defined by quasar coordinates and simultaneously the precise positions of the antennas in the terrestrial frame (Earth-fixed frame, e.g. VTRF08 (Böckmann et al. 2010). Further information about VLBI technology we refer to Campbell $(1979,2004)$, Schuh (1987), Nothnagel (1991), Sovers et al. (1998), Takahashi et al. (2000), Böhm et al. (2011a), Teke et al. (2009), Teke (2011).

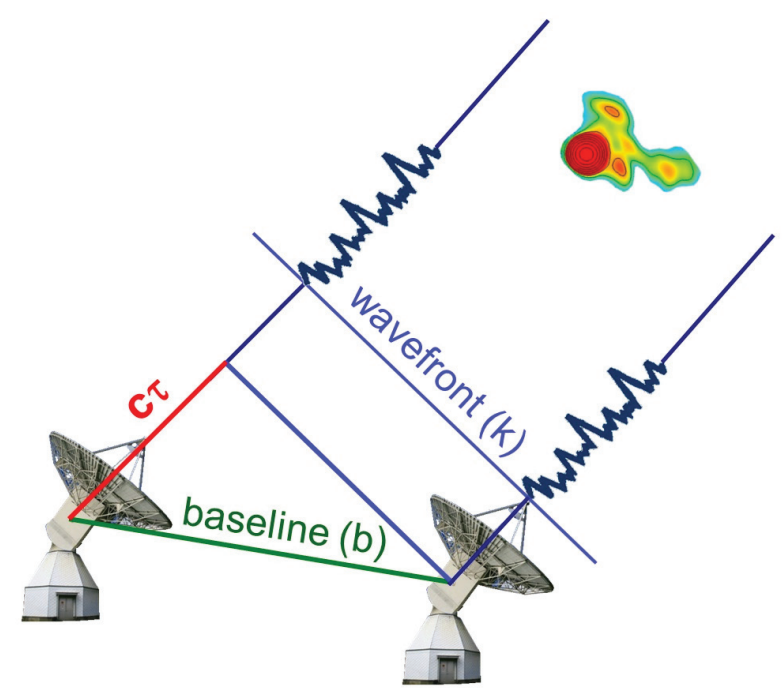

Figure 1: Principle of VLBI observation: The primary observable is the difference in the arrival times of a plane wavefront emitted by an extragalactic radio source at two VLBI telescopes
In Section 2, the VLBI delay model is introduced which is known as "consensus model" and proposed in the International Earth Rotation and Reference Systems Service (IERS) Conventions 2010. We basically follow the IERS Conventions 2010 for the transformation of antenna coordinates from the International Terrestrial Reference System (ITRS) to the Geocentric Celestial Reference System (GCRS) and for the VLBI delay model (Petit and Luzum 2010). Subsection 2.3 and 2.4 reviews the delay models in VLBI analysis due to clock and troposphere errors, respectively. Subsection 2.5 presents the partial derivatives of the VLBI delay model with respect to the estimated parameters are mainly based on Sovers et al. (1998), Böhm et al. (2011a) and Teke (2011). In Section 3, we introduce continuous piece-wise linear offset (CPWLO) functions for sub-daily VLBI parameter estimation.

\section{VLBI Delay Model}

The VLBI observation equation (delay model) can be simply written as

$-c \cdot \tau=\boldsymbol{b}^{\oplus} \cdot \boldsymbol{k}^{\odot}+\Delta \tau_{\text {clock }}+\Delta \tau_{\text {trop }}+\Delta \tau_{\text {iono }}+\Delta \tau_{\text {relativistic }}{ }$

where $c$ is the velocity of light in vacuum environment, $\boldsymbol{k}^{\odot}$ is the unit source (quasar) vector defined in a space-fixed, barycentric and equatorial celestial system, e.g. ICRF2, $\boldsymbol{b}^{\oplus}$ is the baseline vector of the VLBI antennas defined in an Earth-fixed, geocentric, equatorial terrestrial coordinate system, e.g. VTRF08. $\Delta \tau_{\text {clock }}$ is the delay correction due to the synchronization and frequency discrepancies of atomic clocks relative to a fixed clock, $\Delta \tau_{\text {trop }}$ is the troposphere delay correction, $\Delta \tau_{\text {iono }}$ is the ionosphere delay correction and $\Delta \tau_{\text {relativistic }}$ are the delay corrections due to the relativistic effects.

The VLBI delay model is designed primarily for the analysis of VLBI observations to extra-galactic objects from the surface of the Earth. The observable quantities of the VLBI space geodetic technique are recorded signals measured in proper time by the station clocks. The VLBI clocks are synchronized to Universal Time Coordinated (UTC). The ICRF catalogue coordinates of the sources are defined in the Barycentric Celestial Reference System (BCRS) (spacefixed, origin is the center of the mass of the Solar system, equatorial) and the VLBI antennas coordinates are defined in the International Terrestrial Reference System (ITRS) (Earth-fixed, origin is the center of the mass of the Earth). There is another coordinate system defined as Geocentric Celestial Reference System (GCRS) which is kinematically non-rotating w.r.t. BCRS and the origin is at the center of the mass of the Earth. The calculated delay is the time of arrival at station $2, t_{2}$, minus the time of the arrival at station $1, t_{1}$, in GCRS (Petit and Luzum 2010). The UTC time tag of the scan at the first station, i.e. $t_{1}$, serves as the time reference for the measurement and all scalar and vector quantities are assumed to be calculated at $t_{1}$, including e.g. the troposphere delay, tidal corrections on antenna TRF coordinates, the barycentric velocity of the geocenter. However, $t_{1}$ with time scale UTC should be transformed to the appropriate time scale corresponding to the time argument to be used 
to compute each element of the geometric model, e.g. terrestrial time or terrestrial time in Julian centuries since the fundamental (standard) epoch. First, at the time of the observation, the velocity corrections, eccentricities, tidal effects (i.e. solid Earth tides, tidal ocean loading, tidal and non-tidal atmosphere loading, pole tide) are introduced to the VLBI antenna ITRF catalogue coordinates. Thus, the antenna coordinates at the epoch of the scan are derived $\left(\boldsymbol{x}_{\text {ITRS }}\right)$. Then, the transformation to relate the ITRS to GCRS at epoch of observation (scan) can be written as:

$\boldsymbol{x}_{G C R S}=\boldsymbol{Q}(t) \cdot \boldsymbol{R}(t) \cdot \boldsymbol{W}(t) \cdot \boldsymbol{x}_{I T R S}$.

$\boldsymbol{Q}(t), \boldsymbol{R}(t)$, and $\boldsymbol{W}(t)$ are transformation matrices arising from the motion of celestial pole in GCRS (nutation), from the rotation of the Earth around the axis of the pole, and from polar motion in the ITRS, respectively. In Equation (2) the date of the observation $t$ is the equivalence of $t_{1}$ in Terrestrial Time (TT) in Julian centuries since the reference epoch J2000 (2000 January 1, 12h), named as fundamental or standard epoch:

$t=(T T-51544.5) / 36525$

where $T T$ is the Terrestrial Time given by,

$T T=U T C+(T T-T A I)-(U T C-T A I)$,

$T T=t_{1}+(32.184$-leap seconds $) / 86400$.

where $T T-T A I=32.184$ by definition and UTC-TAI refers to the leap seconds (leap seconds is announced as -34 seconds from $1^{\text {st }}$ of January 2009 till $1^{\text {st }}$ of July 2012 by IERS). Regardless from "equinox based" or "Celestial Intermediate Origin (CIO) based" transformation procedures, the transformation matrix arising from polar motion (i.e. relating ITRS and Terrestrial Intermediate Reference System, TIRS) can be expressed as:

$\boldsymbol{W}(t)=\boldsymbol{R}_{3}\left(s^{\prime}\right) \cdot \boldsymbol{R}_{2}\left(x_{p}\right) \cdot \boldsymbol{R}_{1}\left(y_{p}\right)$

where $x_{p}$ and $y_{p}$ being the "polar coordinates" of the Celestial Intermediate Pole (CIP) in the ITRS. $\boldsymbol{R}_{1}, \boldsymbol{R}_{2}$ and $\boldsymbol{R}_{3}$ denote rotation matrices with positive angle about the first, second, and third axes of the Cartesian coordinate system. $s^{\prime}$ is a quantitiy, named "Terrestrial Intermediate Origin (TIO) locater", which provides the position of the TIO on the equator of the CIP corresponding the kinematical definition of the "nonrotating" origin (NRO) in the ITRS at date $t$. The quantity $s^{\prime}$ is sensitive only to the largest variations in polar motion. Using the current mean amplitudes for the Chandlerian and annual wobbles results in (Capitaine et al. 1986; Lambert and Bizouard 2002):

$s^{\prime}=-47 \cdot 10^{-6} \cdot t$.

The CIO based transformation matrix for the Earth rotation around the CIP axis (i.e. relating the TIRS and the Celestial Intermediate Reference System, CIRS) can be expressed as:

$\boldsymbol{R}(t)=\boldsymbol{R}_{3}\left(-E R A\left(t_{u}\right)\right)$

where ERA is the Earth Rotation Angle between the CIO and
TIO at date $t$ on the equator of the CIP, which provides a rigorous definition of the sidereal rotation of the Earth. The $E R A$ can be derived from $U T 1$ as follows:

$E R A\left(t_{u}\right)=2 \pi\left(E R A_{0}+a k \cdot t_{u}\right)$.

In Equation (8), ak is 1.00273781191135448 and $E R A_{0}$ is 0.7790572732640 , and $t_{u}$ is the observation epoch in days since the fundamental reference epoch (UT1-51544.5). UT1 at the observation epoch in $M J D$ can be expressed as

$U T 1=t_{1}+\Delta U T 1 / 86400$

if $\triangle U T 1$ is in seconds. The CIO based transformation matrix arising from the motion of the CIP in the GCRS (i.e. relating CIRS and GCRS), can be expressed as:

$\boldsymbol{Q}(t)=\boldsymbol{R}_{3}(-E) \cdot \boldsymbol{R}_{2}(-d) \cdot \boldsymbol{R}_{3}(E) \cdot \boldsymbol{R}_{3}(s)$.

The quantities $E, d$, and $s$ can be calculated from the coordinates of the CIP in the GCRS as follows:

$$
\begin{aligned}
& E=\arctan \frac{Y}{X}, \\
& d=\arccos Z=\arccos \left(\sqrt{1-X^{2}+Y^{2}}\right), \\
& s=s_{0}-\frac{X Y}{2} .
\end{aligned}
$$

In Equation (10), $s$ is a quantity named "CIO locator", which provides the CIO position on the equator of the CIP corresponding to the kinematical definition of the non-rotating origin (NRO) in the GCRS when the CIP is moving with respect to the GCRS, between the fundamental reference epoch and date $t$ due to precession and nutation (Capitaine et al. 2000, 2002; Petit and Luzum 2010). After having the $\boldsymbol{x}_{G C R S}$ coordinates of the VLBI antennas at the observation epoch $t_{1}, \boldsymbol{x}_{i}^{\oplus}\left(t_{1}\right)$, the gravitational and vacuum delays at the corresponding time are calculated. Detailed explanation on gravitational and vacuum delays and related models are given in subsections 2.1 and 2.2.

\subsection{Gravitational Delay}

The gravitational delay on the signal of the extra-galactic radio source caused by the $J^{\text {th }}$ gravitating body can be computed as,

$$
\Delta T_{\text {grav } J}=2 \frac{G M_{J}}{c^{3}} \ln \frac{\left|\boldsymbol{r}_{1 J}\right|+\boldsymbol{k}^{\odot} \cdot \boldsymbol{r}_{1 J}}{\left|\boldsymbol{r}_{2 J}\right|+\boldsymbol{k}^{\odot} \cdot \boldsymbol{r}_{2 J}},
$$

where $G$ is the Gravitational Constant, $M_{j}$ is the rest mass of the $J^{\text {th }}$ gravitating body, $c$ is the velocity of light, $\boldsymbol{r}_{i J}$ is the vector from the $J^{\text {th }}$ gravitating body to the $i^{\text {th }}$ antenna (receiver), the absolute value sign denotes the norm of the corresponding vector, $|\boldsymbol{x}|=\sqrt{\sum x_{i}^{2}} . k^{\odot}$ is the unit vector from barycenter to the source in the absence of gravitational and aberrational bending,

$\boldsymbol{k}^{\odot}=\left[\begin{array}{lll}\cos \delta \cos \alpha & \cos \delta \sin \alpha & \sin \delta\end{array}\right]$, 
where $\delta$ denotes to declination and $\alpha$ is right ascension defined in the BCRS. The gravitational delay on the signal caused by the Earth can be computed as follows,

$\Delta T_{\operatorname{grav} \oplus}=2 \frac{G M_{\oplus}}{c^{3}} \ln \frac{\left|\boldsymbol{x}_{1}^{\oplus}\right|+\boldsymbol{k}^{\odot} \cdot \boldsymbol{x}_{1}^{\oplus}}{\left|\boldsymbol{x}_{2}^{\oplus}\right|+\boldsymbol{k}^{\odot} \cdot \boldsymbol{x}_{2}^{\oplus}}$,

where $\boldsymbol{x}_{i}^{\oplus}$ denotes the GCRS coordinates of the $i^{\text {th }}$ antenna at epoch $t_{1}$ and $M_{\oplus}$ is the rest mass of the Earth. The $\boldsymbol{r}_{i J}$ vector should be computed at the time $\left(t_{i J}\right)$ when the ray is passing closest to the $J^{\text {th }}$ gravitating body. So the time correction to $t_{1}$ can be computed as follows,

$$
\begin{aligned}
& t_{1 J}=t_{1}-\frac{\boldsymbol{k}^{\odot} \cdot\left(\boldsymbol{x}_{J}^{\odot}\left(t_{1}\right)-\boldsymbol{x}_{1}^{\odot}\left(t_{1}\right)\right)}{c} \\
& \boldsymbol{x}_{J}^{\odot}\left(t_{1 J}\right)=\boldsymbol{x}_{J}^{\odot}\left(t_{1}\right)+\boldsymbol{v}_{J}^{\odot}\left(t_{1 J}-t_{1}\right)
\end{aligned}
$$

where $\boldsymbol{x}_{J}^{\odot}$ and $\boldsymbol{v}_{J}^{\odot}$ denote to the barycentric coordinate and velocity vector of the $J^{\text {th }}$ gravitating body. $\boldsymbol{x}_{J}^{\odot}$ and $t_{1 J}$ can be found by e.g. an iterative approach. The difference vector between gravitating body and the first antenna can be computed as

$$
r_{1 J}\left(t_{1}\right)=\boldsymbol{x}_{1}^{\odot}\left(t_{1}\right)-\boldsymbol{x}_{J}^{\odot}\left(t_{1 J}\right)
$$

where the barycentric coordinates of the $i^{\text {th }}$ antenna $\boldsymbol{x}_{i}^{\odot}$ at epoch $t_{1}$ can be derived from the barycentric coordinates of the geocenter $\boldsymbol{x}_{\oplus}^{\odot}\left(t_{1}\right)$ and GCRS coordinates of the antenna, $\boldsymbol{x}_{i}^{\oplus}\left(t_{1}\right)$ as follows,

$\boldsymbol{x}_{i}^{\odot}\left(t_{1}\right)=\boldsymbol{x}_{\oplus}^{\odot}\left(t_{1}\right)+\boldsymbol{x}_{i}^{\oplus}\left(t_{1}\right)$

and the vector between the gravitating body and the second antenna considering the motion of second antenna during the propagation time between station 1 and station 2 can be derived as follows,

$\boldsymbol{r}_{2 J}=\boldsymbol{x}_{2}^{\odot}\left(t_{1}\right)-\frac{\boldsymbol{v}_{\oplus}^{\odot}}{c}\left(\boldsymbol{k}^{\odot} \cdot \boldsymbol{b}^{\oplus}\right)-\boldsymbol{x}_{J}^{\odot}\left(t_{1 J}\right)$

$\boldsymbol{v}_{\oplus}^{\odot}$ denotes the barycentric velocity vector of the geocenter, and $\boldsymbol{b}^{\oplus}$ is the GCRS baseline vector at $t_{1}$, i.e. $\boldsymbol{x}_{2}^{\oplus}\left(t_{1}\right)-\boldsymbol{x}_{1}^{\oplus}\left(t_{1}\right)$. The total gravitational delay is the sum of all gravitating bodies including the Earth,

$$
\Delta T_{\text {grav }}=\sum_{j} \Delta T_{\text {grav } J}
$$

\subsection{Vacuum Delay and Geometric Delay}

The total geocentric vacuum delay is given by

$$
t_{v 2}-t_{v 1}=\frac{\Delta T_{\text {grav }}-\frac{\boldsymbol{k}^{\odot} \cdot \boldsymbol{b}^{\oplus}}{c}\left[1-\frac{(1+\gamma) \cdot U}{c^{2}}-\frac{\left|\boldsymbol{v}_{\oplus}^{\odot}\right|^{2}}{2 c^{2}}-\frac{\boldsymbol{v}_{\oplus}^{\odot} \cdot \boldsymbol{\omega}_{2}^{\oplus}}{c^{2}}\right]-\frac{\boldsymbol{v}_{\oplus}^{\odot} \cdot \boldsymbol{b}^{\oplus}}{c^{2}}\left(1+\frac{\boldsymbol{k}^{\odot} \cdot \boldsymbol{v}_{\oplus}^{\odot}}{2 c}\right)}{1+\frac{\boldsymbol{k}^{\odot} \cdot\left(\boldsymbol{v}_{\oplus}^{\odot}+\omega_{2}^{\oplus}\right)}{c}}
$$

where $\gamma=1$ according to general relativity using BCRS, $\omega_{i}^{\oplus}$ is the geocentric velocity vector of the $i^{\text {th }}$ antenna, and $U$ denotes to the gravitational potential at the geocenter, neglecting the effects of the Earth's mass,

$U=\frac{G M_{\odot}}{\left|\boldsymbol{r}_{\odot \oplus}\right|}$,

where $\boldsymbol{r}_{\odot \oplus}$, is the vector from the Sun to the geocenter, and $M_{\odot}$ the rest mass of the Sun, $t_{v i}$ is the "vacuum" geocentric coordinate time (TCG) of arrival of a radio signal at the $i^{\text {th }}$ VLBI receiver including the gravitational bending but neglecting the atmospheric delay and atmospheric bending. Some of the vector quantities used in the computation of gravitational and vacuum delays, e.g., $\boldsymbol{x}_{\oplus}^{\odot}, \boldsymbol{v}_{\oplus}^{\odot}, \boldsymbol{x}_{J}^{\odot}, \boldsymbol{v}_{J}^{\odot}$ can be externally provided from planetary ephemerides, e.g. JPL421. The geometric delay can be obtained by adding the geometric part of the troposphere propagation delay to the vacuum delay as follows:

$t_{g 2}-t_{g 1}=t_{v 2}-t_{v 1}+\delta t_{a t m 1} \frac{\boldsymbol{k}^{\odot} \cdot\left(\omega_{2}^{\oplus}-\omega_{1}^{\oplus}\right)}{c}$,

where $\delta_{a t m 1}$ can be the hydrostatic part of the troposphere delay at antenna 1 at observation epoch $t_{1}$ :

$\delta_{\text {atm } 1}=\frac{\Delta L_{h}^{s t(1)}\left(t_{1}\right) \cdot m_{h}^{s t(1)}\left(\varepsilon, t_{1}\right)}{c}$,

where $\Delta L_{h}^{s t(1)}\left(t_{1}\right)$ denotes the troposphere zenith hydrostatic delay at antenna 1 at epoch $t_{1}, m_{h}$ is the hydrostatic mapping function and $\varepsilon$ is the elevation angle. After introducing the corrections which should be applied to the baseline, being elevation and azimuth dependent, i.e. thermal deformation, axis offset correction, and the hydrostatic a priori part of the troposphere delay, the total delay $t_{2}-t_{1}$ can be derived from the geometric delay, $t_{g 2}-t_{g 1}$ as:

$t_{2}-t_{1}=t_{g 2}-t_{g 1}+\tau_{\text {therm deft } 12}+\tau_{\text {axis offset } 12}+\tau_{\text {trop } 12}$

The zenith distance and azimuth of the observation is calculated from the aberrated source vector $\boldsymbol{k}_{i}^{\oplus}$ after transformation to TRF. Thus, the values, e.g. for mapping functions, troposphere gradients, troposphere hydrostatic and wet delays are corrected for aberration. The aberrated source vector can be calculated as follows:

$$
\boldsymbol{k}_{i}^{\oplus}=\boldsymbol{k}^{\odot}+\frac{\boldsymbol{v}_{\oplus}^{\odot}+\omega_{i}^{\oplus}}{c}-\boldsymbol{k}^{\odot} \cdot \frac{\boldsymbol{k}^{\odot} \cdot\left(\boldsymbol{v}_{\oplus}^{\odot}+\omega_{i}^{\oplus}\right)}{c} .
$$

It should be noted that the VLBI observation data include the ionosphere delay (the linear combination of dual frequency measurements is used to calculate the dispersive effect of the ionosphere) in addition to the observed delay. Thus, we assume that the ionosphere delay has already been reduced from the observed delay. The reduced observation (observed minus computed) value ( $o c$ ) for the corresponding observation can be derived as follows:

$o c=\tau_{\text {observed }}-\left(t_{2}-t_{1}\right)$. 
The $\boldsymbol{o c}$ vector basically includes clock error due to synchronization and frequency instabilities, troposphere zenith wet delays, azimuthal asymmetric parts of the troposphere delay, and the effect of the errors in the other a priori values used to calculate $\left(t_{2}-t_{1}\right)$, (e.g. EOP) which all can be estimated in the parameter estimation stage of the VLBI analysis (Petit and Luzum 2010; Böhm et al. 2011a; Teke 2011).

\subsection{Delay Due to Clock Synchronization and Frequency Instability Errors}

In VLBI analysis, the clock errors between two Earth-fixed clocks (mostly hydrogen masers) are estimated by quadratic clock polynomials plus continuous piecewise linear offsets (CPWLO) at pre-determined time intervals with the least squares method (LS), e.g. based on the Gauss-Markof model (Teke 2011):

$$
\Delta \tau_{c l k}(t)=\Delta t_{c l k}^{\text {poly }}(t)+\Delta t_{c l k}^{c p w l o}(t) .
$$

The polynomial for each clock may be formed as

$$
\Delta \tau_{c l k}^{p o l y}(t)=\beta_{0}+\beta_{1}\left(t-t_{0}\right)+\beta_{2}\left(t-t_{0}\right)^{2}
$$

where $t$ is the epoch of the observation in UTC, $t_{0}$ is an arbitrary epoch, e.g. the beginning of the session. $\beta_{j}(j=0,1,2)$ are the polynomial coefficients (offset, rate and quadratic term). The continuous piecewise linear offset (CPWLO) function representation (e.g. for the delay caused by clock errors, $\Delta t_{c l k}^{c p w l o}(t)$ is explained in detail in Section 3.

\subsection{Troposphere Delay}

The troposphere (strictly speaking, the electrically neutral part of the atmosphere) causes a delay on the electromagnetic microwave signals and bending on the ray path when propagating from the source to the receiver. The (slant) delay ( $\Delta L$, which is the notation of the equivalence of $\Delta \tau_{\text {trop }}$ in meters: $\left.\Delta L=\mathrm{c} \cdot \Delta \tau_{\text {trop }}\right)$ along the slant path $(s)$ between the station and the top of the troposphere $\left(H_{\text {trop }}\right)$ can be expressed as the integral over the sum of hydrostatic and wet refractivity $\left(N_{h, w}\right)$ (refractive index, $\left.n\right)$ :

$$
\begin{aligned}
\Delta L & =\int_{s}(n-1) d s=10^{-6} \int_{s} N d s \\
& =10^{-6} \int_{0}^{H_{\text {trop }}}\left[N_{h}(s)+N_{w}(s)\right] d s .
\end{aligned}
$$

Equation (31) can be decomposed into an elevation angle dependent part (spherical part) and an azimuthally asymmetric part known as troposphere gradient (non-spherical part) as follows (Davis et al. 1993):

$\Delta L=\Delta L_{\text {spherical }}(\varepsilon)+\Delta L_{\text {non-spherical }}(\alpha, \varepsilon)$.

The elevation angle dependent part can be written as (Davis et al. 1993)

$$
\Delta L_{\text {spherical }}(\varepsilon)+Z H D m_{h}(\varepsilon)+Z W D m_{w}(\varepsilon)
$$

where $\varepsilon$ is the elevation angle from local horizon, and ZHD the zenith hydrostatic delay, which can be computed from the total pressure $p$ and the station coordinates (latitude $\varphi$ and height $h$ ) with (Saastamoinen 1972):

$$
Z H D=0.0022768 \frac{p}{\left(1-0.00266 \cdot \cos (2 \varphi)-0.28 \cdot 10^{-6} \cdot h\right)}
$$

In Equation (33) $Z W D$ is the zenith wet delay, $m_{h}(\varepsilon)$ and $m_{w}(\varepsilon)$ are the hydrostatic and wet mapping functions which are independent of the azimuth of the observations (e.g.; Niell 1996; Böhm et al. 2006) and which are formulated with the fractional form (Marini 1972):

$$
m_{h, w}(\varepsilon)=\frac{1+\frac{a_{h, w}}{1+\frac{b_{h, w}}{1+c_{h, w}}}}{\sin (\varepsilon)+\frac{a_{h, w}}{\sin (\varepsilon)+\frac{b_{h, w}}{\sin (\varepsilon)+c_{h, w}}}} .
$$

where $a_{h, w}, b_{h, w}$ and $c_{h, w}$ are the hydrostatic and wet coefficients. In Equation (32) the azimuthally asymmetric part can be modeled as (Davis et al. 1993):

$\Delta L_{\text {non-spherical }}(\alpha, \varepsilon)=m_{g}(\varepsilon)\left[G_{n} \cos (\alpha)+G_{e} \sin (\alpha)\right]$

where $\alpha$ is the azimuth (angle from geodetic north), $G_{n}$ and $G_{e}$ are the north and east troposphere total (wet plus hydrostatic) horizontal gradients and $m_{g}(\varepsilon)$ is the gradient mapping function. According to MacMillan (1995), it is

$m_{g}(\varepsilon)=m_{h}(\varepsilon) \cot (\varepsilon)$,

and according to Chen and Herring (1997) it is

$m_{g}(\varepsilon)=\frac{1}{\tan (\varepsilon) \sin (\varepsilon)+C}$

where $C=0.0032$ for the estimation of total gradients (Herring 1992). If Equation (38) is used with the coefficient $C=0.0007$ it describes the gradient mapping function for the wet part and is rather close to the formulation of MacMillan (1995) given in Equation (37) (Böhm et al. 2011b). The troposphere delay model, explained in this section, is widely used when analyzing the observations of the space geodetic techniques which use signals at radio wavelengths, i.e. VLBI, Global Navigation Satellite Systems (GNSS) and Doppler Orbitography and Radio Positioning Integrated by Satellite (DORIS). For most of the VLBI analyses, ZHD are calculated from Equation (34), mapped to the corresponding elevation angles of the observations with a hydrostatic mapping function, e.g. $\mathrm{VMF}_{h}$ (Böhm et al. 2006), and reduced from each observation a priori to the parameter estimation. At the parameter estimation stage of VLBI the estimated tropospheric parameters are $Z W D, G_{n}$ and $G_{e}$ (Teke et al. 2009). 
Troposphere delays are the dominant error source on the observations of space geodetic techniques due to the difficulties of modeling rapidly varying wet refractivity in time and space. Uncertainties in the troposphere delay models propagate into all geodetic estimates, and in particular into the height component of the station positions (Herring 1986; Davis et al. 1991). There is latitude and seasonal dependence of the errors of estimated troposphere delays which can be unveiled through inter-technique comparison of the troposphere estimates derived from the observations of space geodetic techniques i.e. VLBI, GPS, DORIS (Teke et al. 2011).

\subsection{Partial Derivatives of the VLBI Delay Model with respect to Earth Orientation Parameters, Antenna and Source Coordinates}

The partial derivatives of the VLBI delay model w.r.t. a parameter for a session represents the rate with which an observation changes if the corresponding parameter is varied and all the other parameters are kept constant. For the sake of simplicity a basic VLBI delay model for the calculation of partial derivatives w.r.t. the estimated parameters can be used in the parameter estimation. Thus, we do not consider relativistic effects in the delay model for the calculation of partial derivatives. So, the gravitational delay and the transformation of station positions between GCRS and BCRS (Lorentz transformation) due to the center of mass (origin) difference are not considered. Using the partial derivatives of the basic delay model does not produce a difference on the final geodetic estimates comparing to those derived from a complex model. Thus, the simple delay model can be written as follows:

$$
\tau=\boldsymbol{k}^{\odot} \cdot \boldsymbol{Q} \cdot \boldsymbol{R} \cdot \boldsymbol{W} \cdot \boldsymbol{b}^{\oplus} .
$$

In Equation (39), $\boldsymbol{k}^{\odot}$ is the barycentric source vector defined in BCRS, and $\boldsymbol{b}^{\oplus}$ is the geocentric baseline vector defined in TRF. The partial derivative of the simplified VLBI delay model w.r.t. the CIP coordinates in TRF along the Greenwich meridian, $x_{p}$, is:

$$
\frac{\partial \tau}{\partial x_{p}}=\boldsymbol{k}^{\odot} \cdot \boldsymbol{Q} \cdot \boldsymbol{R} \cdot \frac{\partial \boldsymbol{W}}{\partial x_{p}} \cdot \boldsymbol{b}^{\oplus},
$$

where

$$
\frac{\partial \boldsymbol{W}}{\partial x_{p}}=\boldsymbol{R}_{3}\left(s^{\prime}\right) \cdot\left[\begin{array}{ccc}
-\sin x_{p} & 0 & -\cos x_{p} \\
0 & 0 & 0 \\
\cos x_{p} & 0 & -\sin x_{p}
\end{array}\right] \cdot \boldsymbol{R}_{1}\left(y_{p}\right) .
$$

The partial derivative of the delay model w.r.t. the CIP coordinate in TRF along 270 degree east longitude, $y_{p}$, is:

$\frac{\partial \tau}{\partial y_{p}}=\boldsymbol{k}^{\odot} \cdot \boldsymbol{Q} \cdot \boldsymbol{R} \cdot \frac{\partial \boldsymbol{W}}{\partial y_{p}} \cdot \boldsymbol{b}^{\oplus}$,

where

$$
\frac{\partial \boldsymbol{W}}{\partial y_{p}}=\boldsymbol{R}_{3}\left(s^{\prime}\right) \cdot \boldsymbol{R}_{2}\left(x_{p}\right) \cdot\left[\begin{array}{ccc}
0 & 0 & 0 \\
0 & -\sin y_{p} & \cos y_{p} \\
0 & -\cos y_{p} & -\sin y_{p}
\end{array}\right]
$$

The partial derivative of the delay model w.r.t. Earth phase of rotation, $U T 1$, is:

$$
\frac{\partial \tau}{\partial(U T 1)}=\boldsymbol{k}^{\odot} \cdot \boldsymbol{Q} \cdot \frac{\partial \boldsymbol{R}}{\partial(-E R A)} \cdot \frac{\partial(-E R A)}{\partial(U T 1)} \cdot \boldsymbol{W} \cdot \boldsymbol{b}^{\oplus},
$$

where

$$
\frac{\partial \boldsymbol{R}}{\partial(-E R A)}=\left[\begin{array}{ccc}
-\sin (-E R A) & \cos (-E R A) & 0 \\
-\cos (-E R A) & -\sin (-E R A) & 0 \\
0 & 0 & 0
\end{array}\right]
$$

and

$$
\frac{\partial(-E R A)}{\partial(U T 1)}=-a k=-1.00273781191135448 .
$$

The transformation matrix arising from the motion of celestial pole in CRS is composed of four transformation matrices given in Equation (10). The calculation of $E, d$, and $s$ is given in Equations (11, 12, and 13). The partial derivative of the delay model w.r.t. the CIP coordinate $X$ in CRF is:

$$
\frac{\partial \tau}{\partial X}=\boldsymbol{k}^{\odot} \cdot \frac{\partial \boldsymbol{Q}}{\partial X} \cdot \boldsymbol{R} \cdot \boldsymbol{W} \cdot \boldsymbol{b}^{\oplus}
$$

where

$$
\begin{aligned}
\frac{\partial \boldsymbol{Q}}{\partial X}= & \frac{\partial \boldsymbol{R}_{3}(-E)}{\partial(-E)} \cdot \frac{\partial(-E)}{\partial X} \cdot \boldsymbol{R}_{2}(-d) \cdot \boldsymbol{R}_{3}(E) \cdot \boldsymbol{R}_{3}(s) \\
& +\boldsymbol{R}_{3}(-E) \cdot \frac{\partial \boldsymbol{R}_{2}(-d)}{\partial(-d)} \cdot \frac{\partial(-d)}{\partial X} \cdot \boldsymbol{R}_{3}(E) \cdot \boldsymbol{R}_{3}(s) \\
& +\boldsymbol{R}_{3}(-E) \cdot \boldsymbol{R}_{2}(-d) \cdot \frac{\partial \boldsymbol{R}_{3}(E)}{\partial E} \cdot \frac{\partial E}{\partial X} \cdot \boldsymbol{R}_{3}(s) \\
& +\boldsymbol{R}_{3}(-E) \cdot \boldsymbol{R}_{2}(-d) \cdot \boldsymbol{R}_{3}(E) \cdot \frac{\partial \boldsymbol{R}_{3}(s)}{\partial s} \cdot \frac{\partial s}{\partial X}
\end{aligned}
$$

In Equations (48) and (53), $\boldsymbol{R}_{i}(i=1,2,3)$ are the rotation matrices around the first, second, and third axis and $\partial \boldsymbol{R}_{i} / \partial$ are the partial derivatives of the rotation matrices w.r.t. corresponding rotation angles.

$\frac{\partial(-E)}{\partial X}=\frac{Y}{X^{2}+Y^{2}}$,

$\frac{\partial(-d)}{\partial X}=\frac{X}{\sqrt{1-\left(X^{2}+Y^{2}\right)} \sqrt{X^{2}+Y^{2}}}$, 


$$
\frac{\partial s}{\partial X}=\frac{-Y}{2}
$$

The partial derivative of the delay model w.r.t. the CIP coordinate $Y$ in $\mathrm{CRF}$ is:

$$
\frac{\partial \tau}{\partial Y}=\boldsymbol{k}^{\odot} \cdot \frac{\partial \boldsymbol{Q}}{\partial Y} \cdot \boldsymbol{R} \cdot \boldsymbol{W} \cdot \boldsymbol{b}^{\oplus},
$$

where

$$
\begin{aligned}
\frac{\partial \boldsymbol{Q}}{\partial Y}= & \frac{\partial \boldsymbol{R}_{3}(-E)}{\partial(-E)} \cdot \frac{\partial(-E)}{\partial Y} \cdot \boldsymbol{R}_{2}(-d) \cdot \boldsymbol{R}_{3}(E) \cdot \boldsymbol{R}_{3}(s) \\
& +\boldsymbol{R}_{3}(-E) \cdot \frac{\partial \boldsymbol{R}_{2}(-d)}{\partial(-d)} \cdot \frac{\partial(-d)}{\partial Y} \cdot \boldsymbol{R}_{3}(E) \cdot \boldsymbol{R}_{3}(s) \\
& +\boldsymbol{R}_{3}(-E) \cdot \boldsymbol{R}_{2}(-d) \cdot \frac{\partial \boldsymbol{R}_{3}(E)}{\partial E} \cdot \frac{\partial E}{\partial Y} \cdot \boldsymbol{R}_{3}(s) \\
& +\boldsymbol{R}_{3}(-E) \cdot \boldsymbol{R}_{2}(-d) \cdot \boldsymbol{R}_{3}(E) \cdot \frac{\partial \boldsymbol{R}_{3}(s)}{\partial s} \cdot \frac{\partial s}{\partial Y}
\end{aligned}
$$

In Equation (53),

$\frac{\partial(-E)}{\partial Y}=\frac{-X}{X^{2}+Y^{2}}$

$\frac{\partial(-d)}{\partial Y}=\frac{Y}{\sqrt{1-\left(X^{2}+Y^{2}\right)} \sqrt{X^{2}+Y^{2}}}$,

$$
\frac{\partial s}{\partial Y}=\frac{-X}{2}
$$

The partial derivative of the delay model w.r.t. right ascension of a source is:

$$
\frac{\partial \tau}{\partial \alpha}=\frac{\partial \boldsymbol{k}^{\odot}}{\partial \alpha} \cdot \boldsymbol{Q} \cdot \boldsymbol{R} \cdot \boldsymbol{W} \cdot \boldsymbol{b}^{\oplus}
$$

where

$$
\frac{\partial \boldsymbol{k}^{\odot}}{\partial \alpha}=\left[\begin{array}{lll}
-\cos \delta \sin \alpha & \cos \delta \cos \alpha & 0
\end{array}\right] .
$$

The partial derivative of the delay model w.r.t. declination of a source is:

$$
\frac{\partial \tau}{\partial \delta}=\frac{\partial \boldsymbol{k}^{\odot}}{\partial \delta} \cdot \boldsymbol{Q} \cdot \boldsymbol{R} \cdot \boldsymbol{W} \cdot \boldsymbol{b}^{\oplus},
$$

where

$$
\frac{\partial \boldsymbol{k}^{\odot}}{\partial \delta}=\left[\begin{array}{lll}
-\sin \delta \cos \alpha & -\sin \delta \sin \alpha & \cos \delta
\end{array}\right]
$$

The partial derivative of the delay model w.r.t. the TRF coordinate of the antennas are e.g. for the $X$ component of station 1 :

$$
\frac{\partial \tau}{\partial X_{s t 1}}=\boldsymbol{k}^{\odot} \cdot \boldsymbol{Q} \cdot \boldsymbol{R} \cdot \boldsymbol{W} \cdot \frac{\partial \boldsymbol{b}^{\oplus}}{\partial X_{s t 1}},
$$

where

$$
\frac{\partial \boldsymbol{b}^{\oplus}}{\partial X_{s t 1}}=\left[\begin{array}{l}
1 \\
0 \\
0
\end{array}\right] .
$$

As an example, the partial derivatives of the delay model w.r.t. Earth phase of rotation, UT1 given in Equation (44) are calculated for three baselines observing two sources for a 24 hour VLBI session carried out on $16^{\text {th }}$ of August 2008. This example shows Kokee (Hawaii, USA) - Wettzell (Germany) baseline is east-west oriented with a length of about 10360 $\mathrm{km}$, Westford (USA) - Wettzell baseline is also east-west oriented (6000 km), Onsala (Sweden) - Wettzell baseline is north-south oriented $(920 \mathrm{~km})$. When Kokee and Wettzell VLBI stations observe the source $1357+769$ at about $9^{\text {h }}$ and $21^{\mathrm{h}} U T C$ on $16^{\text {th }}$ of August 2008 one millisecond (ms) variation in $U T 1$ results in $\sim 15 \mathrm{~cm}$ change on the corresponding observation i.e. on the delay (red circles, Figure 2). However, at about $3^{\mathrm{h}}$ and $15^{\mathrm{h}}$ UTC the UT1 change does nearly not affect the corresponding delay, because $U T 1$ is sensitive to the observing baseline rotation in space w.r.t. the observed source when the corresponding baseline is east-west oriented.

\section{Continuous Piece-wise Linear Offset (CPWLO) Functions for Sub-daily Parameter Estimation}

Similar to continuous piece-wise linear (CPWL) functions where the initial offset and rates are formed, we directly estimate offsets at every UTC integer hours or fractions of integer hours (sub-daily parameter estimation) with the continuous piece-wise linear offset (CPWLO) functions (Böhm et al. 2011a; Teke 2011) given below:

$x_{i}=x_{1}+\frac{t-t_{1}}{t_{2}-t_{1}}\left(x_{2}-x_{1}\right)$

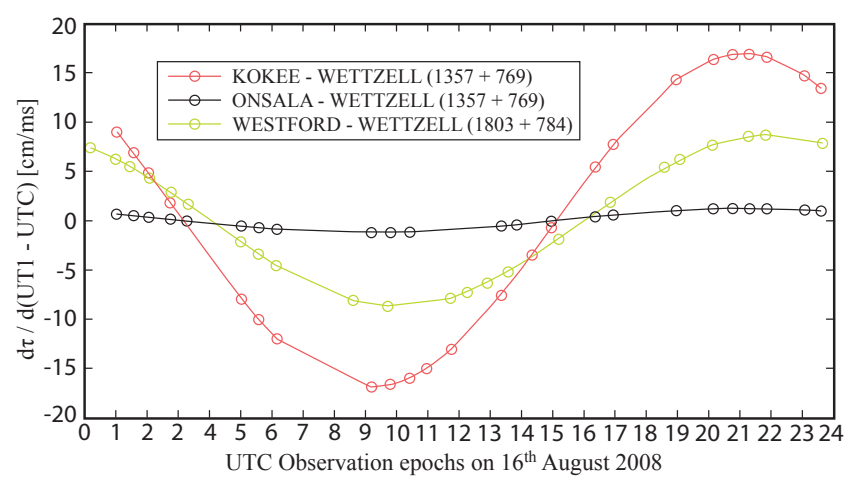

Figure 2: The partial derivatives of the delay model w.r.t. UT1 for three baselines: Kokee-Wettzell, Onsala-Wettzell and Westford-Wettzell observing two polar sources: $1357+769$ and 1803+784 for a 24 hour VLBI session carried out on $16^{\text {th }}$ of August 2008. 
In the above function $x_{1}$ and $x_{2}$ are CPWLO estimated at integer UTC hours, fractions or multiples of UTC integer hours $\left(t_{1}\right.$ and $\left.t_{2}\right) . t$ is the epoch (observation epoch) of the functional value, $x_{i}$. The illustration of a CPWLO function can be seen in Figure 3.

In VLBI analysis clock errors, zenith wet delays, troposphere gradients, Earth orientation parameters, TRF and CRF coordinates can be estimated as CPWLO. The partial derivatives of the delay model w.r.t. an unknown parameter, $x_{i}$ at the observation epoch $t$ can be derived as follows:

$$
\begin{gathered}
\frac{\partial \tau(t)}{\partial x_{1}}=\frac{\partial \tau(t)}{\partial x_{i}} \cdot \frac{\partial x_{i}}{\partial x_{1}}, \\
\frac{\partial \tau(t)}{\partial x_{2}}=\frac{\partial \tau(t)}{\partial x_{i}} \cdot \frac{\partial x_{i}}{\partial x_{2}},
\end{gathered}
$$

where the partial derivatives of the CPWLO function given in Equation (63) w.r.t. its first and second offsets, $x_{1}$ and $x_{2}$, are:

$$
\begin{aligned}
& \frac{\partial x_{i}}{\partial x_{1}}=1-\frac{t-t_{j}}{t_{j+1}-t_{j}}, \\
& \frac{\partial x_{i}}{\partial x_{2}}=\frac{t-t_{j}}{t_{j+1}-t_{j}} .
\end{aligned}
$$

It should be noted that the observation epoch, $t$, should fulfill the condition $t_{j}<t<t_{j+1}$ for CPWLO functions. In case, we want to derive the partial derivative of the delay model w.r.t. $U T 1$ at epoch $t$, the partials can be derived for two unknown CPWLO (one estimation interval) as follows:

$$
\begin{gathered}
\frac{\partial \tau(t)}{\partial x_{1(U T 1)}}=\frac{\partial \tau(t)}{\partial(U T 1)} \cdot \frac{\partial(U T 1)}{\partial x_{1}}, \\
\frac{\partial \tau(t)}{\partial x_{2(U T 1)}}=\frac{\partial \tau(t)}{\partial(U T 1)} \cdot \frac{\partial(U T 1)}{\partial x_{2}} .
\end{gathered}
$$

In Equations (68) and (69) $\partial \tau(t) / \partial(U T 1)$ is the partial derivative of the delay model at the observation epoch $t$ w.r.t. $U T 1$ given in Equation (44), $\partial(U T 1) / \partial x_{1,2}$ are the partial

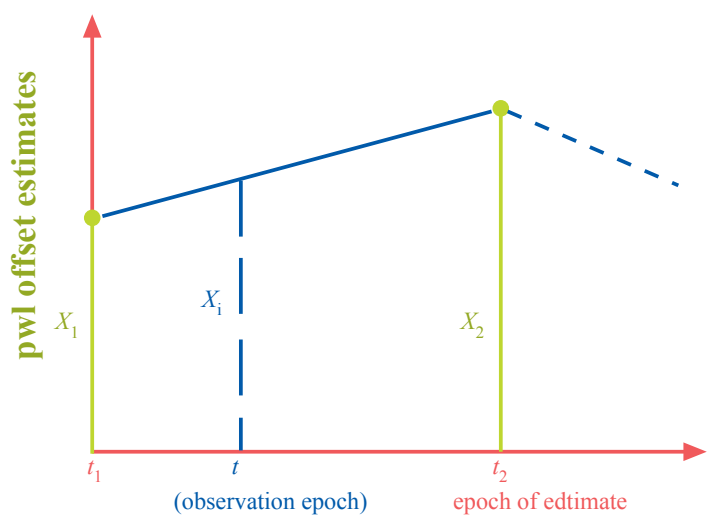

Figure 3: Continuous piece-wise linear offsets (CPWLO). derivatives of $U T 1$ w.r.t. its first and second offset as provided in Equations (66) and (67). In case, we want to derive the partial derivative of the delay model w.r.t. the two CPWLO TRF coordinates of antenna $1, s t 1$, e.g. for the $X$ component, we use:

$$
\begin{gathered}
\frac{\partial \tau(t)}{\partial x_{1\left(X_{s t 1}\right)}}=\frac{\partial \tau(t)}{\partial X_{s t 1}} \cdot \frac{\partial X_{s t 1}}{\partial x_{1}} \\
\frac{\partial \tau(t)}{\partial x_{2\left(X_{s t 1}\right)}}=\frac{\partial \tau(t)}{\partial X_{s t 1}} \cdot \frac{\partial X_{s t 1}}{\partial x_{2}}
\end{gathered}
$$

In Equations (70) and (71) $\partial \tau(t) / \partial X_{s t 1}$ is the partial derivative of the delay model at the observation epoch $t$ w.r.t. the $X$ coordinate of antenna 1 as written in Equation (61), and $\partial X_{s t 1} / \partial x_{1,2}$ are the partial derivatives of the antenna coordinates w.r.t. its first and second offsets as given in Equations (66) and (67).

As an example, the design matrix consisting of TRF antenna coordinates ( $X$ components) for three antennas and three observations carried out at the epochs $t 1^{\prime}, t 2^{\prime}$, and $t 3^{\prime}$ and one estimation interval (two CPWLO unknowns for each antenna) can be written as:

$$
\left[\begin{array}{cccccc}
\frac{\partial \tau\left(t 1^{\prime}\right)}{\partial x_{1\left(X_{s t 1}\right)}} & \frac{\partial \tau\left(t 1^{\prime}\right)}{\partial x_{2\left(X_{s t 1}\right)}} & \frac{-\partial \tau\left(t 1^{\prime}\right)}{\partial x_{1\left(X_{s t 2}\right)}} & \frac{-\partial \tau\left(t 1^{\prime}\right)}{\partial x_{2\left(X_{s t 2}\right)}} & 0 & 0 \\
0 & 0 & \frac{\partial \tau\left(t 2^{\prime}\right)}{\partial x_{1\left(X_{s t 2}\right)}} & \frac{\partial \tau\left(t 2^{\prime}\right)}{\partial x_{2\left(X_{s t 2}\right)}} & \frac{-\partial \tau\left(t 2^{\prime}\right)}{\partial x_{1\left(X_{s t 3}\right)}} & \frac{-\partial \tau\left(t 2^{\prime}\right)}{\partial x_{2\left(X_{s t 3}\right)}} \\
\frac{\partial \tau\left(t 3^{\prime}\right)}{\partial x_{1\left(X_{s t 1}\right)}} & \frac{\partial \tau\left(t 3^{\prime}\right)}{\partial x_{2\left(X_{s t 1}\right)}} & 0 & 0 & \frac{-\partial \tau\left(t 3^{\prime}\right)}{\partial x_{1\left(X_{s t 3}\right)}} & \frac{-\partial \tau\left(t 3^{\prime}\right)}{\partial x_{2\left(X_{s t 3}\right)}}
\end{array}\right]
$$

To demonstrate the good agreement of troposphere zenith delays CPWLO estimates between VLBI and other space geodetic techniques at a co-located site i.e. Wettzell, Figure 4 is shown.

The CPWLO zenith total delay estimates obtained with the Vienna VLBI Software (VieVS) (Böhm et al. 2011a) developed at Vienna University of Technology, Institute of Geodesy and Geophysics are in a very good agreement with the Bernese (Dach et al. 2007) GPS solution of Center for Orbit Determination in Europe (CODE) (Dach et al. 2009) within $5 \mathrm{~mm}$ standard deviation. The High Resolution Limited Area Model (HIRLAM) (Undén et al. 2002), a numerical weather model covering Europe, is in a rather good agreement with other space geodetic techniques i.e. GPS and VLBI with a standard deviation of about $15 \mathrm{~mm}$.

\section{Conclusions}

The geodetic parameters such as coefficients of the clock model, troposphere zenith delays, troposphere gradients (delays due to the azimuthally asymmetric part of the troposphere), Earth orientation parameters (polar motion coordinates, UT1-UTC, nutation offsets), VLBI antenna reference point coordinates in the TRF, and radio source coordinates in the CRF can be simultaneously and accurately estimated 


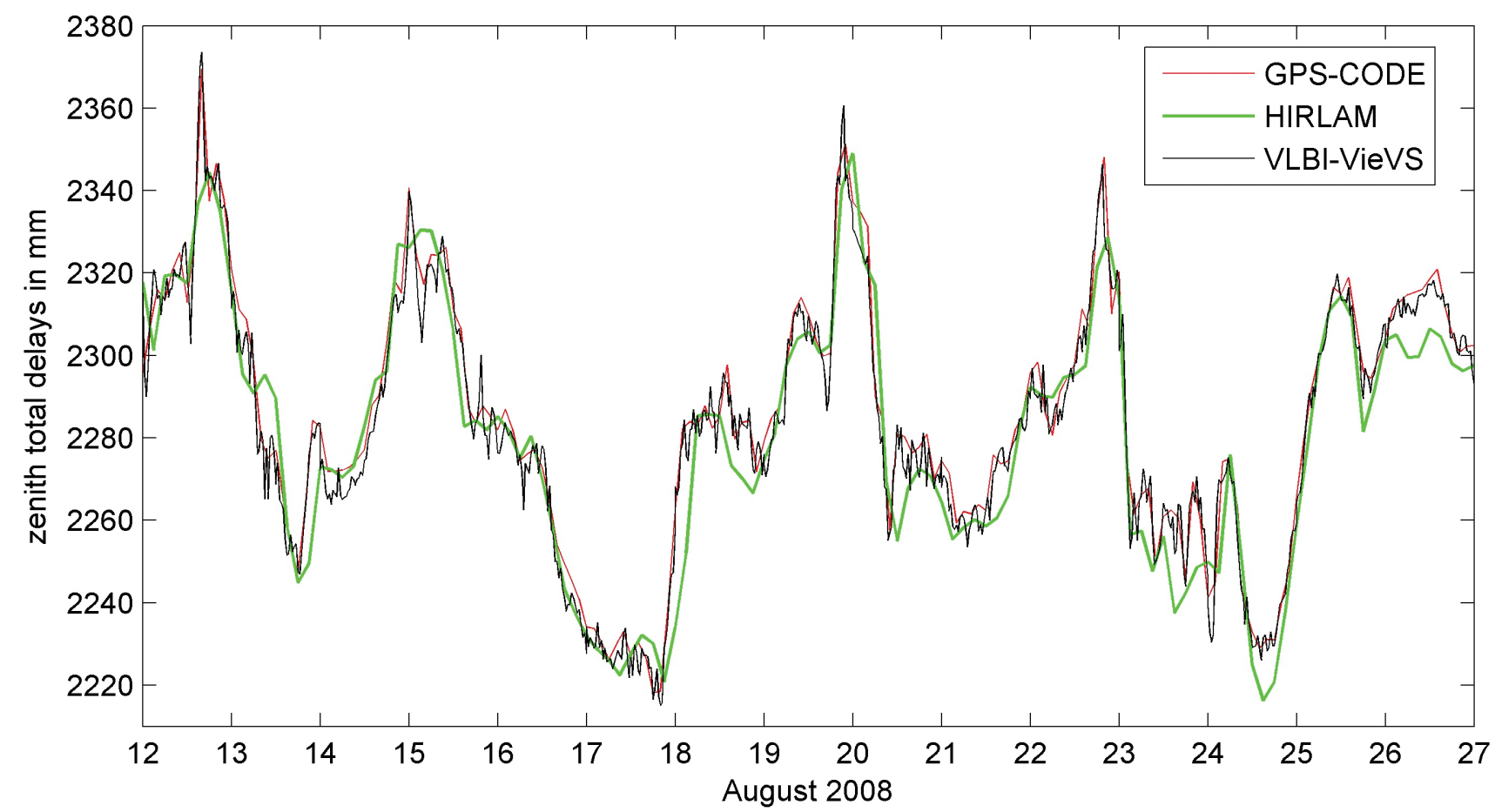

Figure 4: Continuous piece-wise linear offset (CPWLO) estimates of troposphere zenith total delays at co-located site Wettzell (Germany) during CONT08. CONT08 sessions were carried out during 12 - 27 August 2008 with 11 co-located sites on five continents by the International VLBI Service for Geodesy and Astrometry (IVS). GPS-CODE is the troposphere solution of one of the global IGS Analysis Centers, Center for Orbit Determination in Europe (CODE). HIRLAM, is the troposphere model product of the numerical weather model, High Resolution limited Area Model for Europe. VLBIVieVS is the troposphere solution of VLBI analysis software, Vienna VLBI Software (VieVS) which is a state-of-the-art software developed at Vienna University of Technology, Institute of Geodesy and Geophysics.

from VLBI observations. If enough observations with a homogenous distribution w.r.t. time and space are carried out for each estimation interval, sub-daily resolution of the parameters can be achieved with continuous piecewise linear offset (CPWLO) functions.

\section{Acknowledgements}

We use in this study the data provided by the International VLBI Service for Geodesy and Astrometry (IVS). The authors would like to acknowledge one of the anonymous reviewers for his constructive and very useful remarks on the paper.

\section{References}

Böckmann S., Artz T., Nothnagel A., (2010), VLBI terrestrial reference frame contributions to ITRF2008, J. Geod., 84, 201-219, doi:10.1007/s00190-009-0357-7.

Böhm J., Böhm S., Nilsson T., Pany A., Plank L., Spicakova H., Teke K., Schuh H., (2011a), The new Vienna VLBI software VieVS, In: International Association of Geodesy Symposia Series, (Kenyon S., Pacino M.C., Marti U., Eds.), Vol. 136, pp. 1007-1013.

Böhm J., Spicakova H., Urquhart L., Steigenberger P., Schuh H., (2011b), Impact of a priori gradients on VLBI-Derived terrestrial reference frames, In: Proceedings of the $20^{\text {th }}$ Meeting of the European VLBI Group for Geodesy and Astronomy, (Alef W., Bernhart S., Nothnagel A., Eds.), Institut für Geodäsie und Geoinformation, Universität Bonn, Germany, pp.128-132.
Böhm J., Werl B., Schuh H., (2006), Troposphere mapping functions for GPS and Very Long Baseline Interferometry from European Center for Medium-Range Weather Forecasts operational analysis data, J. Geophys. Res., 111(B02406).

Campbell J., (2004), VLBI for Geodesy and Geodynamics, In: The Role of VLBI in Astrophysics, Astrometry and Geodesy, (Mantovani F., Kus A., Eds.), Kluwer Academic Publishers, pp.359-381.

Campbell J., (1979), Die Radiointerferometrie auf langen Basen als geodätisches Messprinzip hoher Genauigkeit, DGK Reihe C, Heft 254, Verlag des Instituts für Angewandte Geodäsie, Frankfurt am Main.

Capitaine N., Gambis D., McCarthy D., Petit G., Ray J., Richter B., Rothacher M., Standish M., Vondrak J., (2002), Proceedings of the IERS Workshop on the Implementation of the New IAU Resolutions, IERS Technical Note 29, Verlag des Bundesamts für Kartographie und Geodäsie, Frankfurt am Main.

Capitaine N., Guinot B., McCarthy D., (2000), Definition of the celestial ephemeris origin and of UT1 in the international celestial reference frame, Astron. Astrophys., 335(1), 398-405.

Capitaine N., Guinot B., Souchay J., (1986), A non-rotating origin on the instantaneous equator: Definition, properties and use, Celest. Mech., 39(3), 283-307.

Chen G., Herring T., (1997), Effects of atmospheric azimuthal asymmetry on the analysis from space geodetic data, J. Geophys. Res., 102 (B9), 20489-20502.

Dach R., Brockmann E., Schaer S., Beutler G., Meindl M., Prange L., Bock H., Jäggi A., Ostini L., (2009), GNSS processing at CODE: status report, J. Geod., 83, 353-365.

Dach, R., Hugentobler U., Fridez P., Meindl M., (2007), Bernese GPS Software Version 5.0., Switzerland: Astronomical Institute, University of Bern. 
Davis J., Herring T., Shapiro I., (1991), Effects of atmospheric modeling errors on determinations of baseline vectors from VLBI, J. Geophys. Res., 96 (B1), pp. 643-650.

Davis J., Elgered G., Niell A.E., Kuehn C.E., (1993), Ground-based measurements of gradients in the "wet" radio refractivity of air, Radio Sci., 28(6), 1003-1018.

Fey A., Gordon D., Jacobs C.S., (2009), The second realization of the international celestial reference frame by very long baseline interferometry, IERS Technical Note; 35 . Verlag des Bundesamts für Kartographie und Geodäsie, Frankfurt am Main, ISBN 3-89888-918-6, 204pp.

Herring T., (1992), Modeling atmospheric delays in the analysis of space geodetic data, In: Proceedings of Refraction of Transatmospheric Signals in Geodesy, (DeMunck J.C., Spoelstra T.A., Eds.), Netherlands Geodetic Comission Series, Publications on Geodesy, Vol.36, pp.157-164.

Herring T., (1986), Precision of vertical estimates from very long baseline interferometry, J. Geophys. Res., 91(B9), 9177-9182.

Lambert S., Bizouard C., (2002), Positioning the terrestrial ephemeris origin in the international terrestrial reference frame, Astron. Astrophys, 394(1), 317-321.

MacMillan D., (1995), Atmospheric gradients from very long baseline interferometry observations, J. Geophys. Res. Lett., 22(9), 1041-1044.

Marini J., (1972), Correction of satellite tracking data for an arbitrary tropospheric profile, Radio Sci., 7(2), 223-231.

Niell A., (1996), Global mapping functions for the atmosphere delay at radio wavelengths, J Geophys. Res., 101(B2), 3227 3246.

Nothnagel A., (1991), Radiointerferometrische Beobachtungen zur Bestimmung der Polbewegung unter Benutzug langer NordSüd-Basislinien, DGK Reihe C, Heft 368, Verlag des Instituts für Angewandte Geodäsie, Frankfurt am Main.

Petit G., Luzum B., (2010), IERS Conventions 2010, IERS Technical Note ; 36, Frankfurt am Main: Verlag des Bundesamts für Kartographie und Geodäsie, ISBN 3-89888-989-6, 179 pp.
Saastamoinen J., (1972), The use of artificial satellites for geodesy, In: Geophys. Monogr. Ser., Vol.15, pp.247-251, American Geophysical Union, Washington.

Schlüter W., Behrend D., (2007), The International VLBI Service for Geodesy and Astrometry (IVS): Current capabilities and future prospects, J. Geod., 81(6-8), 379-387.

Schuh H., (1987), Die Radiointerferometrie auf langen Basen zur Bestimmung von Punktverschiebungen und Erdrotationsparametern, DGK Reihe C, Heft 328, Verlag der Bayerischen Akademie der Wissenschaften, München.

Sovers O.J., Fanselow J.L., Jacobs C.S., (1998), Astrometry and geodesy with radio interferometry: Experiments, models, results, Reviews of Modern Physics, 70(4).

Takahashi F., Kondo T., Takahashi Y., Koyama Y., (2000), Wave Summit Course: Very Long Baseline Interferometer, Published by Ohmsha, Ltd., ISBN: 1-58603-076-0 (IOS Press), Tokyo.

Teke K., (2011), Sub-daily parameter estimation in VLBI data analysis, Geowissenschaftliche Mitteilungen, Heft Nr. 87, Vienna University of Technology, Vienna, Austria. Grafisches Zentrum HTU GmbH, ISSN 1811-8380.

Teke K., Böhm J., Nilsson T., Schuh H., Steigenberger P., Dach R., Heinkelmann R., Willis P., Haas R., Espada S.G., Hobiger T., Ichikawa R., Shimizu S., (2011), Multi-technique comparison of troposphere zenith delays and gradients during CONT08, J. Geod., 85(7), 395-413.

Teke K., Boehm J., Tanır E., Schuh H., (2009), Çok Uzun Baz Enterferometrisi (VLBI) tekniğinde, saat hatast ve troposferik gecikme parametre kestirim modelleri, Harita Dergisi, 142, 1-16.

Undén, P., Rontu L., Järvinen H., Lynch P., Calvo J., Cats G., Cuxart J., Eerola K., Fortelius C., Garcia-Moya J., Jones C., Lenderlink G., McDonald A., McGrath R., Navascues B., Woetman N., Ødegaard V., Rodriguez E., Rummukainen M., Room R., Sattler K., Hansen S., Savijärvi H., Wichers S., Sigg R., The H., Tijm A., (2002), HIRLAM-5 Scientific Documentation, Technical report, Swedish Meteorological and Hyrdological Institute, Norrköping. 